\title{
THE VERY LONG BASELINE ARRAY
}

\author{
PETER J. NAPIER \\ National Radio Astronomy Observatory, P.O.Box 0, Socorro, NM 87801, \\ USA
}

\begin{abstract}
The Very Long Baseline Array, currently under construction, is a dedicated VLBI array consisting of ten $25 \mathrm{~m}$ diameter antennas and a 20 station correlator. The key design features that make it a flexible, general purpose instrument are reviewed and some details are provided of the array layout, frequency coverage and sensitivity.
\end{abstract}

\section{INTRODUCTION}

Construction of the Very Long Baseline Array (VLBA) began in 1985 and is scheduled for completion in 1992. The goal of the project is to provide an instrument which can carry out research on a wide range of problems in the field of very high resolution radio astronomy. General information about the VLBA and its potential research areas is available in Kellerman and Thompson (1985) and a summary of its instrumental parameters is provided by Vanden Bout (1991). In this paper we briefly review the current major design goals for the instrument and give some details of the array layout and antenna and receiver performance. Details of other aspects of the VLBA can be found elsewhere in these proceedings; see Rogers (tape recorder), Bagri and Thompson (electronics design), Thompson and Bagri (calibration system) and Chikada (correlator).

\section{PROJECT DESIGN GOALS}

The principal design goals for the VLBA are as follows:

(1) Provide an array of 10 identical elements dedicated to VLBI astronomy. The antennas will be in continuous operation under remote control from the Array Operations Center (AOC) in Socorro. The intent is to make the operation of the array and its correlator sufficiently reliable and "user friendly" so that VLBI techniques will be readily available to all astronomers.

(2) The locations of the elements of the array will be chosen to provide the highest possible resolution and best uv-plane coverage possible with ten antennas located on US territory.

(3) The feeds and receivers will provide frequency flexibility. Nine receiver bands in the range $327 \mathrm{MHz}$ to $43 \mathrm{GHz}$ will be provided with several of the receivers having bandwidths of a few tens of percent to allow multi-frequency 
synthesis (Biretta et al. in these proceedings). A dual frequency feed for the $2.3 / 8.4 \mathrm{GHz}$ bands will be provided for geodesy and astrometry observations. All feeds and receivers will be dual circularly polarized. The antennas will be built with sufficient precision so that an $86 \mathrm{GHz}$ observing capability can be added in the future.

(4) The tape recorders will permit continuous operation at wide bandwidth. Normal operation will be $128 \mathrm{Mbit} / \mathrm{sec}$ with a tape change once per day. With more frequent tape changes a limited number of observations requiring high sensitivity will be able to use 256 or $512 \mathrm{Mbit} / \mathrm{sec}$.

(5) The correlator, to be located at the AOC in Socorro, will be large and highly flexible. It will process up to 20 stations simultaneously at playback rates up to $256 \mathrm{Mbit} / \mathrm{sec}$ with up to 1024 channels of spectral resolution. All polarization products will be available.

\section{ARRAY LAYOUT}

The locations of the 10 VLBA stations are given in Table I and are shown schematically in Figure 1. In late 1990 the antennas are complete, or almost complete, at all sites except St.Croix and Hawaii where the antenna foundations are in preparation. Figure 1 also shows the uv-coverage for this ten element array. It is expected that for a useful amount of time (in the range 10 to $20 \%$ ) the phased VLA and Greenbank telescopes will be added to the VLBA elements to provide increased sensitivity, although without significantly improving the uv-coverage shown in Figure 1. Addition of one or two elements in Europe or South America significantly improves the uv-coverage.

TABLE I VLBA Station Locations

\begin{tabular}{llcrr}
\hline Code & \multicolumn{1}{c}{ Location } & $\begin{array}{c}\text { N Latitude } \\
{[\% \text { ' }]}\end{array}$ & $\begin{array}{c}\text { W Longitude } \\
{\left[0^{\prime \prime \prime}\right]}\end{array}$ & $\begin{array}{c}\text { Elevation } \\
{[\mathrm{m}]}\end{array}$ \\
\hline SC & St. Croix VI & 174530.57 & 643502.61 & 16 \\
HN & Hancock NH & 425600.96 & 715911.69 & 309 \\
NL & N. Liberty IA & 414617.03 & 913426.35 & 241 \\
FD & Fort Davis TX & 303805.63 & 1035639.13 & 1615 \\
LA & Los Alamos NM & 354630.33 & 1061442.01 & 1967 \\
PT & Pie Town NM & 341803.61 & 1080707.24 & 2371 \\
KP & Kitt Peak AZ & 315722.39 & 1113642.26 & 1916 \\
OV & Owens Valley CA & 371354.19 & 1181633.98 & 1207 \\
BR & Brewster WA & 480752.80 & 1194055.34 & 255 \\
MK & Mauna Kea HI & 194815.85 & 1552728.95 & 3720 \\
\hline \hline
\end{tabular}

\section{ANTENNA AND RECEIVER PERFORMANCE}

The VLBA $25 \mathrm{~m}$ diameter antennas use a shaped Cassegrain feed system in which the feeds are located in a circle around the axis of the primary reflector. 
Rapid frequency changing is performed by rotating the subreflector to point to the desired feed. Additionally, for the $327 / 610 \mathrm{MHz}$ bands, a dual frequency crossed-dipole feed is permanently located in the center of the subreflector and is positioned at the prime focus by moving the whole subreflector in focus. The dual frequency capability for $2.3 / 8.4 \mathrm{GHz}$ is provided quasi-optically using a tilted, flat, dichroic reflector over the $2.3 \mathrm{GHx}$ feed and an ellipsoid reflector over the $8.4 \mathrm{GHz}$ feed. The dichroic reflector allows $2.3 \mathrm{GHz}$ signals to pass through to the $2.3 \mathrm{GHz}$ feed and reflects the $8.4 \mathrm{GHz}$ signals to the ellipsoid which focuses them into the $8.4 \mathrm{GHz}$ feed. All frequency changes can be accomplished under computer control in less than $30 \mathrm{sec}$.
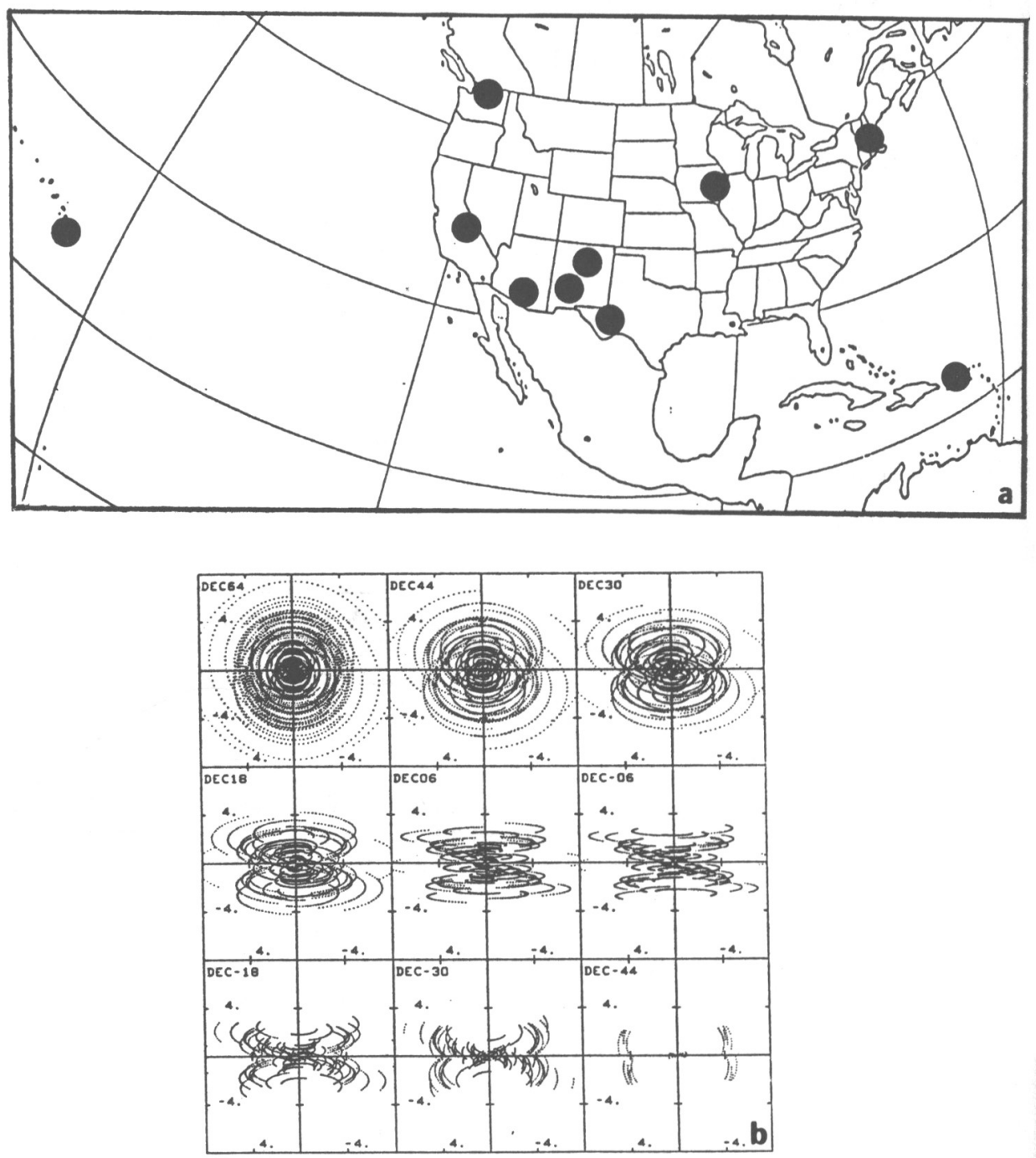

Fig. 1. (a) Location of the ten VLBA stations. (b) uv-plane coverage for these ten stations (baseline units $10^{3} \mathrm{~km}$ ). 
Table II shows typical feed and receiver performance at the zenith measured on an antenna or, for the $327 / 610 \mathrm{MHz}, 14 \mathrm{GHz}$ and $43 \mathrm{GHz}$ receivers for which testing has not yet been completed, predicted values. For $2.3 \mathrm{GHz}$ numbers include the effect of losses in the dichroic reflector which is permanently in position over the feed. For $8.4 \mathrm{GHz}$ the ellipsoid can be moved out of position so performance is given with (denoted DF) and without the dual frequency system in place. The fractional bandwidths given are calculated for the listed frequency range. Tests show (Biretta et al. in these proceedings) that significantly wider bandwidths are available if some loss in sensitivity is accepted. For example, with a loss of a factor of two in sensitivity (aperture efficiency/ $T_{s y s}$ ) compared to the values in Table II, fractional bandwidths available with the $20 \mathrm{~cm}, 6 \mathrm{~cm}$ and $1 \mathrm{~cm}$ receivers are $44 \%, 16 \%$ and $22 \%$ respectively. Finally, preliminary tests on the Pie Town antenna at $86 \mathrm{GHz}$ (Walker and Bagri, 1989) gave an aperture efficiency of $18 \%$ which suggests that useful performance can be achieved at this frequency in the future.

TABLE II VLBA Antenna and Receiver Performance

\begin{tabular}{lcccc}
\hline $\begin{array}{c}\text { Band } \\
\text { Designation }\end{array}$ & $\begin{array}{c}\text { Frequency } \\
\text { Range (GHz) }\end{array}$ & $\begin{array}{c}\text { Fractional } \\
\text { Bandwidth (\%) }\end{array}$ & $\begin{array}{c}\text { Aperture } \\
\text { Efficiency (\%) }\end{array}$ & $\begin{array}{c}\text { Total } \\
T_{\text {sys }}(K)\end{array}$ \\
\hline $90 \mathrm{~cm}$ & $0.312-0.342$ & 9 & 40 & 160 \\
$50 \mathrm{~cm}$ & $0.580-0.640$ & 10 & 40 & 120 \\
$20 \mathrm{~cm}$ & $1.35-1.75$ & 26 & 57 & 32 \\
$13 \mathrm{~cm}$ & $2.15-2.35$ & 9 & 58 & 34 \\
$6 \mathrm{~cm}$ & $4.6-5.1$ & 10 & 72 & 40 \\
$4 \mathrm{~cm}$ & $8.0-8.8$ & 10 & 70 & 35 \\
$4 \mathrm{~cm}(\mathrm{DF})$ & $8.0-8.8$ & 10 & 66 & 41 \\
$2 \mathrm{~cm}$ & $12.1-15.4$ & 24 & 69 & 80 \\
$1 \mathrm{~cm}$ & $21.7-24.1$ & 11 & 62 & 90 \\
$7 \mathrm{~mm}$ & $41-45$ & 9 & 51 & 127 \\
\hline \hline
\end{tabular}

Pointing tests show that the antenna is meeting its pointing specification of 8 arc.sec. rms for short timescale disturbances (wind up to $6 \mathrm{~m} / \mathrm{sec}$ ) and 14 arc.sec. rms for long duration disturbances (thermal). Tests with inclinometers mounted at the elevation axis show that when the antenna is heated on one side by the sun it tilts by approximately 20 arc.sec. peak-to-peak.

\section{ACKNOWLEDGEMENTS}

A large number of scientists and engineers have participated in the design and construction of the VLBA. The principal designers have documented their work in the Project Book (NRAO, 1988) from which much of the information in this paper is taken. In particular I thank C. Walker for the information on array layout and C. Walker and D. Bagri for measurements of antenna efficiency and system temperature. 


\section{REFERENCES}

Kellerman, K. I. K. and Thompson, A. R. 1985, Science, 229, 123.

NRAO 1988,VLBA Project Book, Version 7.

Vanden Bout, P.A. 1991, in Advances in Space Research, in press.

Walker, C. and Bagri, D. 1989, VLBA Memo 656, NRAO.

R. Simon: Can you comment on the expected availability of the Greenbank Telescope (GBT) for VLBA observing?

P. Napier: The Greenbank Telescope is being designed to have the frequency flexibility necessary to make observing with the VLBA easy. Also, a VLBA compatible Data Acquisition System is planned for the telescope. The fraction of time allocated on the GBT for VLBI observing will depend on how competitive the VLBI science proposals are compared to the other science pursued by the GBT.

R. Norris: You mentioned that the plan is to use Internet as the primary communication path for monitor and control data between the AOC in Socorro and the ten VLBA sites. Do you have figures for the reliability of Internet and do you have back-up communications planned?

P. Napier: The current plan is to use the NSF Internet backbone and subnetworks on either side of it to communicate from the AOC to the University Internet node nearest to a remote VLBA site. Discussions with the managers of the backbone and subnetworks give predictions of $98-99 \%$ uptime for this part of the VLBA communication path. The other equally important part is the leased telephone line from the nearby University to the remote VLBA site. The reliability of this phone line will be highly site dependent and may dominate the reliability statistics at some sites. A backup communication path will be provided by making a long-distance dial-up connection direct from the $\mathrm{AOC}$ to the remote site. In the event that phone communication is lost either at the AOC or the remote site, the site operating system will keep the antenna observing for several hours.

G. Hennessy: Do you think it is feasible to use NSFNET/Internet to get correlated data (VLBA or VLA) to the astronomer at their home Institute? P. Napier: Internet is already being used to export VLA databases from the AOC to remote users. Database size must be limited so as not to overload the Internet spur coming in to Socorro. If the intent of the question was to request a remote "real-time" observing capability for the VLA and VLBA, then wideband data links connecting the AOC to the VLA Site and the ten VLBA sites are needed. Such wideband links are being studied for the future but no definite plan, or source of funding, for implementation is available. 